\title{
Nonlinear Wave Propagation Phenomena in Left-Handed Transmission-Line Media
}

\author{
Alexander B. Kozyrev and Daniel W. van der Weide, Member, IEEE
}

\begin{abstract}
This paper introduces nonlinear transmission lines based on left-handed (LH) media and simulates harmonic generation and parametric generation in a material that, in two dimensions, could also focus microwaves. This paper discusses physical phenomena that lead to and affect self-supporting harmonic generation and parametric generation in LH nonlinear transmission-line media and outline advantages of these media for developing new types of compact and efficient frequency multipliers and "active lens" devices.
\end{abstract}

Index Terms-Frequency conversion, left-handed (LH) media, metamaterials, negative refractive index, nonlinear transmission line (NLTL), parametric generation.

\section{INTRODUCTION}

A RTIFICIAL materials (metamaterials) with simultaneously negative permeability and permittivity are also known as left-handed (LH) materials. LH materials often use arrays of metallic wires and arrays of split-ring resonators [1] or planar transmission lines periodically loaded with series capacitors and shunt inductors [2], [3]. The unique electrodynamic properties of these materials, first predicted by Veselago in 1968 [4], include the reversal of Snell's law, the Doppler effect, Cherenkov radiation, and they exhibit a negative refractive index, making them attractive for new types of RF and microwave components. The most tantalizing of these properties is the possibility of realizing "perfect" (diffraction-free) lenses based on their inherent negative index of refraction [5], [3].

The majority of studies of LH media to date are in the linear regime of wave propagation and have already inspired new types of microwave devices, such as LH phase shifters [6], LH directional couplers [7], and leaky-wave antennas [8], [9]. However, materials that combine nonlinearity with the anomalous dispersion exhibited by LH media can give rise to many new and interesting phenomena and applications [10], [11]. Nonlinear wave phenomena that occur during propagation along the boundary between right-handed (RH) and LH media, when one or both of them are nonlinear, have been previously reported in [12]-[15].

In [16], we briefly discussed nonlinear wave phenomena in the LH material alone, as opposed to wave phenomena that occur along an LH to RH media interface. Here, we present a thorough investigation of the basic nonlinear wave propagation phenomena in LH medium, which is based on the dual of the

Manuscript received April 21, 2004. This work was supported by the Air Force Office of Scientific Research under the Multiuniversity Research Initiative Grant F49620-03-1-0420.

The authors are with the Electrical and Computer Engineering Department, University of Wisconsin-Madison, Madison, WI 53705 USA (e-mail: abkozyrev@wisc.edu; danvdw@engr.wisc.edu).

Digital Object Identifier 10.1109/TMTT.2004.839305
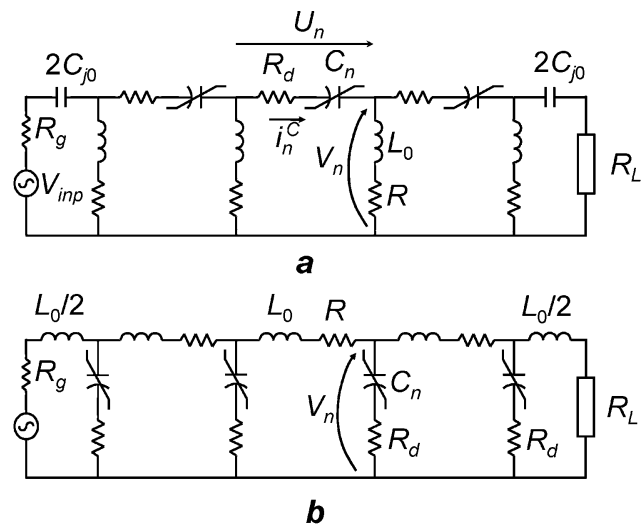

Fig. 1. Equivalent circuit of: (a) LH NLTL and (b) dual RH NLTL.

conventional nonlinear transmission line (NLTL); an LH NLTL with anomalous dispersion. These phenomena include the generation of higher harmonics, parametric wave interaction, and the generation of fractional harmonics. In Section III, we develop an analytical technique for the analysis of harmonic generation in discrete transmission-line systems and calculate the third harmonic conversion efficiency for the circuit model of the LH NLTL described in Section II. Section IV presents the results of simulations of harmonic generation and parametric generation and discusses the mechanism of self-supported quasi-phase matching, which leads to efficient third harmonic generation. A comparison of LH and RH NLTLs configured as frequency multipliers is presented and the advantages of LH NLTLs for providing higher per-stage third harmonic conversion efficiency are outlined. Additionally, the competition between harmonic generation, parametric generation, and the generation of fractional frequencies is discussed. Lastly, in Section V, we discuss the possibility of the implementation of hybrid LH NLTLs useful for applications such as frequency multipliers and parametric generators and amplifiers.

\section{MODEL DESCRIPTION}

Consider a one-dimensional (1-D) LH NLTL circuit shown in Fig. 1(a), which is the dual of a conventional RH NLTL shown in Fig. 1(b). In the linear case, $C_{n}=C_{j 0}$ (capacitance per section), and in the zero loss case, $R_{d}=R=0\left(R_{d}\right.$ and $R$ are the resistances connected in series with capacitance and inductance, respectively), the dispersion relationship is defined as

$$
\omega^{2}=\frac{1}{4 L_{0} C_{j 0} \sin ^{2} \beta / 2}
$$

where $\beta$ is the phase shift (in radians) per section and $L_{0}$ is the inductance per section. The value $\beta=\pi$ corresponds to 
the boundary of the transparency band (the minimal frequency of the propagating wave, also known as the Bragg cutoff frequency)

$$
\omega_{B}=\frac{1}{2 \sqrt{L_{0} C_{j 0}}} .
$$

In the linear limit, the $\mathrm{LH}$ circuit under consideration is a high-pass filter. When $\omega$ is increased, $\beta$ decreases monotonically (anomalous dispersion), thus, the fundamental wave $\left(-\pi \leq \beta_{0} \leq \pi\right)$ propagating in this structure is a backward one (i.e., the phase and group velocities are directed in opposite directions). This property permits us to refer to this structure as LH (in Veselago's terminology [4]).

\section{ANAlytical CONSIDERATion OF ThiRd HaRmonic GENERATION IN LH NLTL}

Let us first consider harmonic generation in LH NLTLs analytically. The approach developed in [17] is applied to derive an analytical expression for the amplitude of the third harmonic generated in discrete LH NLTLs.

Nonlinear wave processes in LH NLTLs are governed by Kirchhoff's laws, stated here as follows:

$$
\begin{aligned}
\frac{d Q_{n+1}}{d t}-\frac{d Q_{n}}{d t}+G\left(U_{n+1}-U_{n}\right) & =i_{n}^{L} \\
L_{0} \frac{d i_{n}^{L}}{d t} & =V_{n}-i_{n}^{L} R .
\end{aligned}
$$

Here, $V_{n}$ and $i_{n}^{L}$ are the voltage at the $n$th node and current through the $n$th inductor, $U_{n}=V_{n}-V_{n-1}$ is the voltage across the $n$th nonlinear capacitor, and $Q_{n}$ is the charge stored in the $n$th capacitor. In order to simplify the analytical expressions in this section, we account for the effect of resistance $R_{d}$ with a conductance $G$, connected in parallel with the capacitors $C_{n}$ (not shown in Fig. 1). This produces an equivalent circuit when the specific frequency represented by the fundamental excitation is used in the circuit model.

It is assumed that the capacitance $C_{n}$ possesses a symmetric nonlinear voltage-charge relationship that can be expanded in a convergent Taylor's series

$$
Q_{n}\left(U_{n}\right)=Q^{\prime}(0) U_{n}+\frac{1}{3 !} Q^{\prime \prime \prime}(0) U_{n}^{3}+\cdots
$$

where $Q^{\prime}(0)=C_{j 0}$ since $d Q_{n}\left(U_{n}\right)=C_{n}\left(U_{n}\right) d U_{n}$.

One can expand $U_{n}$ and $Q_{n}$ in a complex Fourier series as follows:

$$
\begin{gathered}
U_{n}(t)=\sum_{m=1}^{\infty}\left\{U_{m, n} e^{j m \omega t}+U_{m, n}^{*} e^{-j m \omega t}\right\}, \\
m=1,3,5 \ldots
\end{gathered}
$$

and

$$
\begin{gathered}
Q_{n}(t)=\sum_{m=1}^{\infty}\left\{Q_{m, n} e^{j m \omega t}+Q_{m, n}^{*} e^{-j m \omega t}\right\}, \\
m=1,3,5 \ldots
\end{gathered}
$$

where $\omega$ is the lowest frequency sinusoidal component of $U_{n}$ and $Q_{n}$. Eliminating $i_{n}^{L}$ between (3) and (4) and substituting (6) and (7) into the resulting differential-difference equation yields (after equating the terms)

$$
\begin{aligned}
& \left(-m^{2} \omega^{2}+j m \omega R / L_{0}\right)\left(Q_{m, n+1}-2 Q_{m, n}+Q_{m, n-1}\right) \\
& \quad+\left(j m \omega G+R G / L_{0}\right)\left(U_{m, n+1}-2 U_{m, n}+U_{m, n-1}\right) \\
& \quad-U_{m, n} / L_{0}=0, \quad \text { for } m=1,3,5 \ldots
\end{aligned}
$$

Equation (8) represents a system of coupled linear difference equations relating the $U_{m, n}$ and $Q_{m, n}$ coefficients. By substituting (6) and (7) into (5), and making the assumption that harmonic voltage terms are small compared with $U_{1, n}$, one finds that, to third order, these coefficients are also related by

$$
\begin{aligned}
& Q_{1, n}=Q^{\prime}(0) U_{1, n} \\
& Q_{3, n}=Q^{\prime}(0) U_{3, n}+\frac{1}{3 !} Q^{\prime \prime \prime}(0) U_{1, n}^{3} .
\end{aligned}
$$

Thus, the $Q_{m, n}$ coefficients can be systematically eliminated between (8) and (9). For $m=1$ and 3, this procedure leads to

$$
U_{1, n+1}-2 U_{1, n}+U_{1, n-1}-\kappa_{1}^{2} U_{1, n}=0
$$

and

$$
U_{3, n+1}-2 U_{3, n}+U_{3, n-1}-\kappa_{3}^{2} U_{3, n}=-\frac{Q^{\prime \prime \prime}(0) U_{1, n}^{3}}{3 !\left(1-\tan \delta_{3}\right)}
$$

where

$$
\kappa_{m}^{2}=\left(j m \omega L_{0}+R\right)^{-1}\left(G+j m \omega Q^{\prime}(0)\right)^{-1}
$$

and

$$
\delta_{3}=\tan ^{-1}\left(G / 3 \omega Q^{\prime}(0)\right) .
$$

Equation (10) is the homogeneous discrete wave equation for the fundamental frequency voltage $U_{1, n}$. This fundamental voltage wave serves as the "forcing function" for the third harmonic voltage wave $U_{3, n}$ according to (11). Assuming, for simplicity, that only the positive-traveling fundamental wave is excited, the solution to (10) has the form

$$
U_{1, n}=U_{1,0} \exp \left(-\gamma_{1} n\right)
$$

where $n$ is the section number. By substituting (14) into (11), and solving the resultant inhomogeneous difference equation for boundary condition $U_{3,0}=U_{3, \infty}=0$, one obtains the third harmonic amplitude

$$
U_{3, n}=\frac{4 K_{N} U_{1,0}^{3} \sinh ^{2}\left(3 \gamma_{1} / 2\right) e^{-\gamma_{3} n}\left(e^{\left(\gamma_{3}-3 \gamma_{1}\right) n}-1\right)}{\left(1-\tan \delta_{3}\right)\left(\kappa_{3}^{2}-4 \sinh ^{2}\left(3 \gamma_{1} / 2\right)\right)}
$$

where $K_{N}$ is a "nonlinearity factor" defined by

$$
K_{N}=\frac{Q^{\prime \prime \prime}(0)}{3 ! Q^{\prime}(0)}
$$




$$
\gamma_{m}=\alpha_{m}+j \beta_{m}=2 \sinh ^{-1} \frac{\kappa_{m}}{2} .
$$

Assuming relatively small losses $G \ll 3 \omega Q^{\prime}(0)$ and $R \ll$ $3 \omega L_{0}$, one finds the following expression for the third harmonic generation efficiency:

$$
\eta_{3}^{\mathrm{LH}}(n)=\frac{\left|V_{3, n}\right|^{2}}{\left|V_{1,0}\right|^{2}}=K_{N}^{2}\left|U_{1,0}\right|^{4} F_{A}\left(\beta_{1}, \beta_{3}\right) \cdot F_{C}(\phi) \cdot e^{-2 \alpha_{3} n} .
$$

Here, $F_{A}\left(\beta_{1}, \beta_{3}\right)$ is a function that depends on the dispersion characteristics only and determines a maximal conversion efficiency for a given frequency, and $F_{C}(\phi)$ is a periodic in space function, which we call the "coherence function" by analogy with [17]. $F_{A}$ and $F_{C}$ are given by (19) and (20)

$$
\begin{aligned}
F_{A}\left(\beta_{1}, \beta_{3}\right) & =\frac{\sin ^{2} \frac{\beta_{1}}{2}}{\sin ^{2} \frac{\beta_{3}}{2}}\left(\frac{\sin ^{2} \frac{3 \beta_{1}}{2}}{\sin ^{2} \frac{\beta_{3}}{2}-\sin ^{2} \frac{3 \beta_{1}}{2}}\right)^{2} \\
F_{C}(\phi) & =\sin ^{2} \frac{\phi}{2} \\
\phi & =\left(\beta_{3}-3 \beta_{1}\right) n .
\end{aligned}
$$

In (18), we took into account that

$$
U_{m, n}=V_{m, n}\left(1-e^{-j \beta_{m}}\right) .
$$

Applying the technique described above to the RH NLTL, shown in Fig. 1(b), one can show that the expression for $\eta_{3}^{\mathrm{RH}}(n)$ has exactly the same structure as (18) derived for the LH NLTL, where $F_{A}$ becomes

$$
F_{A}\left(\beta_{1}, \beta_{3}\right)=F_{A}^{\mathrm{RH}}\left(\beta_{1}, \beta_{3}\right)=\left(\frac{\sin ^{2} \frac{\beta_{3}}{2}}{\sin ^{2} \frac{3 \beta_{1}}{2}-\sin ^{2} \frac{\beta_{3}}{2}}\right)^{2}
$$

and $\beta_{1}, \beta_{3}$, and $\alpha_{3}$ are determined by the dispersion equation for the RH NLTL

$$
\begin{aligned}
\alpha_{m}+j \beta_{m}= & 2 \sinh ^{-1} \\
& \times\left\{\frac{\left(j m \omega L_{0}+R\right)^{1 / 2}\left(G+j m \omega Q^{\prime}(0)\right)^{1 / 2}}{2}\right\} .
\end{aligned}
$$

Fig. 2 compares $F_{A}$ and the magnitude of the phase mismatch $\left|\beta_{3}-3 \beta_{1}\right|$ for both an LH and RH NLTL. Although the analytic expressions for the third harmonic generation efficiency in both RH and LH NLTLs have a similar structure, the qualitative picture of nonlinear wave processes, in general, and of third harmonic generation, in particular, have many distinctions in LH NLTLs versus the RH NLTL case. These differences are the consequences of the anomalous dispersion exhibited by $\mathrm{LH}$ NLTLs.

Third harmonic generation is possible at $\omega>\omega_{B}$ in the case of LH NLTLs and at $0 \leq \omega \leq \omega_{B}^{\mathrm{RH}} / 3$ in the case of RH NLTLs, where $\omega_{B}^{\mathrm{RH}}=2 /\left(L_{0} C_{j 0}\right)^{1 / 2}$ is the Bragg frequency in the $\mathrm{RH}$

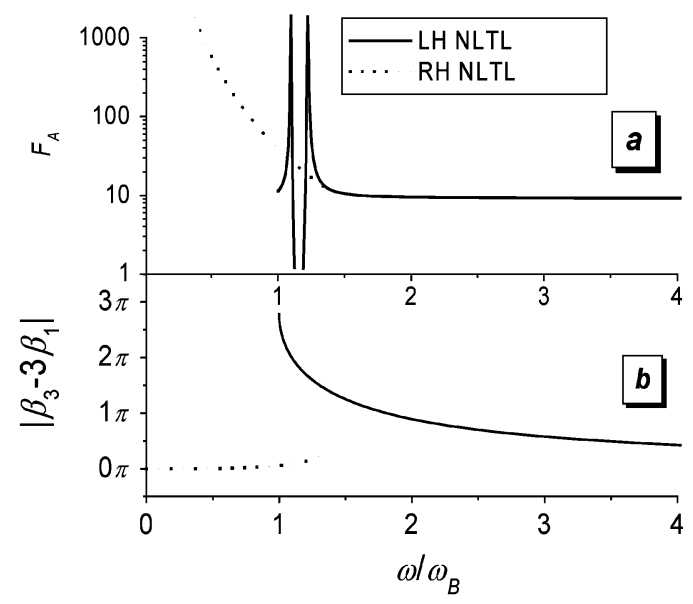

Fig. 2. (a) Dependence of the function $F_{A}$ and (b) magnitude of the phase mismatch $\left|\beta_{3}-3 \beta_{1}\right|$ on the relative fundamental frequency for both LH NLTL (solid line) and RH NLTL (dotted line).

NLTL. Thus, in the case of LH NLTLs, third harmonic generation is possible in a higher and a wider frequency range than in RH NLTLs, with all other parameters being the same (see also Fig. 2).

In the case of RH NLTLs, the value of $\left|\beta_{3}-3 \beta_{1}\right|$ is small in the operating frequency range and tends toward zero when the operating frequency goes to zero $(\omega \rightarrow 0)$. Meanwhile, $F_{A}^{\mathrm{RH}} \rightarrow \infty$ so that the combined effect of functions $F_{A}^{\mathrm{RH}}$ and $F_{C}(\phi)$ is a linear growth, and the optimal length of the NLTL (i.e., the length required to achieve the maximum value of conversion efficiency) is determined by the tradeoff between this linear growth and the exponential decay due to loss along the line [17]. In the case of an LH NLTL, $\left|\beta_{3}-3 \beta_{1}\right|$ is large [see Fig. 2(b)] and so the period of the "coherence function" is short as is the "coherence length" of the interaction of the fundamental wave with its third harmonic, which is defined as

$$
N_{c}=\frac{2 \pi}{\left|\beta_{3}-3 \beta_{1}\right|}
$$

for consistency with [17]. This gives rise to a highly localized energy exchange between the fundamental wave and its third harmonic while propagating along an LH NLTL. From (20), it is apparent that the maximum amplitude of the third harmonic is achieved when $F_{C}(\phi)$ is a maximum at the end of the line, therefore, the optimal number of LH NLTL sections is

$$
N_{\text {opt }}=\frac{(2 k+1) \pi}{\beta_{3}-3 \beta_{1}}, \quad k=0,1,2,3 \ldots
$$

Analysis of (18)-(20) indicates that, despite the large phase mismatch in LH NLTLs, the conversion efficiency can be higher in the case of LH NLTLs versus RH NLTLs for the case of short NLTLs. Additionally, the effect of loss can be minimized as well, again, relative to the RH NLTL case.

Furthermore, Fig. 2 shows that there are two frequencies (wavenumbers) when the denominator in (19) vanishes and $F_{A}\left(\beta_{1}, \beta_{3}\right)$ goes to infinity. At these frequencies, (14)-(20) are no longer valid since our approach assumes that $\left|V_{3, n}\right| \ll\left|V_{1, n}\right|$. Most importantly however, these frequencies 
indicate regions that are potentially promising for efficient harmonic generation.

Thus, the analysis of the function $F_{A}\left(\beta_{1}, \beta_{3}\right)$ together with the magnitude of the phase mismatch $\left|\beta_{3}-3 \beta_{1}\right|$ (or determined by phase mismatch "coherence length" $N_{c}$ ) predicts that the third harmonic generation in short LH NLTLs can be more efficient than in RH NLTL of the same line length. Although it gives a clear qualitative scenario of the harmonic generation, our analysis is not valid for the most interesting practical case of strong nonlinearity (i.e., a large capacitance ratio) and large amplitude. To account for all the factors that affect wave propagation phenomena in an LH NLTL, we have carried out detailed computer simulations. The modeling results are presented in Section IV.

Lastly, we emphasize that the analytical approach introduced here in the example of third harmonic generation can be easily applied to either generation of any higher harmonics or generation of fractional frequencies.

\section{Results of SimUlations}

\section{A. Parameters of LH NLTL Used in Simulations}

It is assumed that the nonlinear capacitors $C_{n}=C\left(V_{n}-\right.$ $\left.V_{n-1}\right)$ are formed by two back-to-back varactor diodes to provide symmetric capacitance-voltage characteristics, which can be expressed as

$$
C(V)=\frac{C_{j 0}}{\left(1+\left|V / V_{j 0}\right|\right)^{M}}
$$

The values for the prototype Agilent TC803 hyperabrupt varactor diodes $C_{j 0}=1 \mathrm{pF}, M=1.039, V_{j 0}=0.7 \mathrm{~V}$, and $R_{d}=$ $6 \Omega$ are used. These diodes were chosen because of their high capacitance ratio $C(0 \mathrm{~V}) / C(10 \mathrm{~V})=17$. The input port of the LH NLTL is fed by a sinusoid $V_{\text {inp }}=V_{0} \sin (\omega t)$. The amplitude of the input is chosen such that the voltage drop across the diodes does not exceed the breakdown voltage $V_{\mathrm{br}}=10 \mathrm{~V}$. The circuit is loaded with resistance $R_{L}=0.8 Z_{0}\left(Z_{0}=\left(L_{0} / C_{j 0}\right)^{1 / 2}\right)$, which is equal to the generator resistance $R_{g}=R_{L}$ (unless the otherwise specified). Simple five- and seven-section LH NLTLs were simulated with Agilent ADS.

\section{B. Third Harmonic Generation}

Fig. 3 plots the conversion efficiency for the generation of the third and fifth harmonics (i.e., the ratio of the harmonic power delivered to the load to the input power applied to LH NLTL) for both the LH NLTL and dual RH NLTL. The modeling results indicate that the generation of higher harmonics is possible within a limited frequency range.

Fig. 4 shows the dependence of $|\phi|$ [defined by (20)] at the end of a five-section LH NLTL $\left(|\phi|=5\left|\beta_{3}-3 \beta_{1}\right|\right)$ on the fundamental frequency. For this purpose, the total phase shift of the fundamental wave and its third harmonic over the entire $\mathrm{LH}$ NLTL has been calculated from the data generated employing computer modeling. One can see that the maximum third harmonic generation efficiency at $\omega / \omega_{B} \approx 2.9$ corresponds to the maximum of the "coherence function" at the output of the LH $\operatorname{NLTL}(|\phi|=5 \pi)$, while the local minima at $\omega / \omega_{B} \approx 2.4$ and $\omega / \omega_{B} \approx 3.9$ corresponds to the "coherence function" minimum

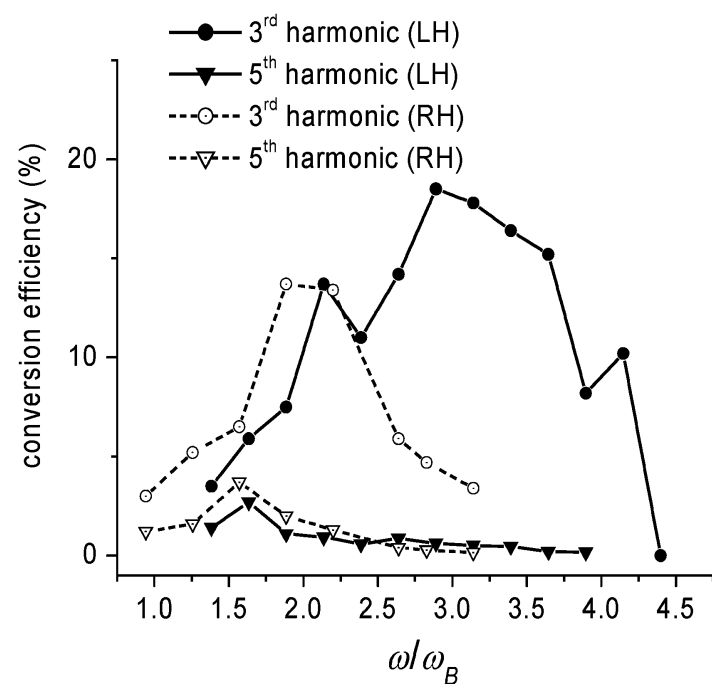

Fig. 3. Conversion efficiency (\%) for the third and the fifth harmonics versus the relative fundamental frequency for a five-section LH NLTL (solid lines) and dual five-section RH NLTL (dashed lines).

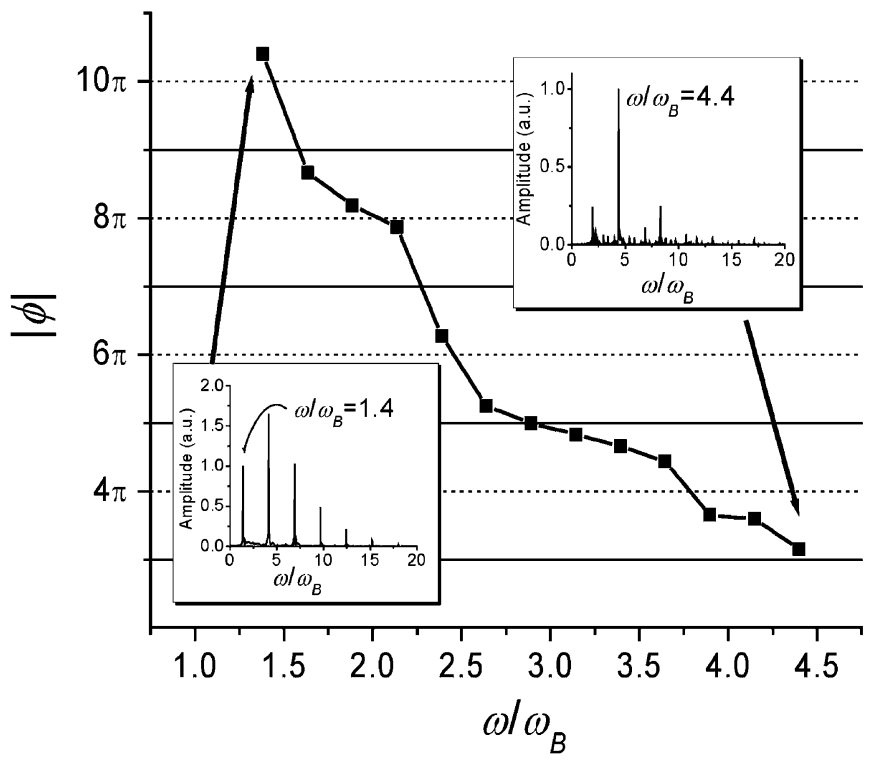

Fig. 4. Dependence of $|\phi|$ at the output of a five-section LH NLTL $\left(|\phi|=5\left|\beta_{3}-3 \beta_{1}\right|\right)$ on the relative fundamental frequency corresponding to the conversion efficiency data shown in Fig. 3. Insets show the spectra of the voltage waveform at the output load for the fundamental frequency $\omega_{\text {inp }} / \omega_{B}=1.4$ and 4.4 , corresponding to the edges of the frequency range under consideration.

$(|\phi|=6 \pi$ and $4 \pi$, respectively). Another local maximum of efficiency near $\omega / \omega_{B} \approx 2.2$ corresponds to the range where the denominator of function $F_{A}$ vanishes. The "coherence function" in this range is near its minimum as well, thus ultimately restricting conversion efficiency.

On the lower frequency side, the third harmonic generation region is bounded by the transmission-line Bragg cutoff frequency (2). The lower left inset in Fig. 4 corresponds to this case. It shows the spectrum of the voltage waveform at the output load of the LH NLTL. A fundamental frequency of $\omega / \omega_{B}=1.4$ generated numerous higher harmonics and the third harmonic dominates over the fundamental and the other higher harmonics due to the intensive Bragg reflection of the 
fundamental wave. Thus, LH NLTLs, under certain conditions, may combine the properties of both a harmonic generator and a bandpass filter and provide a "pure" third harmonic at the output of LH NLTL.

A detailed analysis indicates that, within the frequency range $2.9 \leq \omega / \omega_{B} \leq 3.7$, where the third harmonic conversion efficiency is maximum and $\left|\beta_{3}-3 \beta_{1}\right| \approx \pi$, the amplitude of the voltage oscillations across the nonlinear capacitors varies periodically from section to section. The period of this variation is equivalent to two sections of the LH NLTL. The amplitude is large $\left(U_{\max } \sim U_{\mathrm{br}}\right)$ across odd-spaced capacitors and small $\left(U_{\max } \sim U_{\mathrm{br}} / 5\right)$ across even-spaced capacitors. This fact correlates well with the period of the "coherence function," which requires that the third harmonic amplitude is maximal across even sections and close to zero across odd ones.

This self-induced periodicity of the voltage amplitude across the nonlinear capacitors leads to a periodic variation of the capacitance along the line. Due to the strong nonlinearity (large capacitance ratio), this periodicity results in a considerable change of the dispersion characteristics and enables quasi-phase matching of the fundamental wave and its third harmonic. This would otherwise be impossible in the linear limit (our analytic formula (18) does not take this effect into account). Thus, we have the possibility of self-supporting third harmonic generation over a wide frequency range. This self-induced quasi-phase-matching effect allows the amplitude of the third harmonic, at the points corresponding to the maximum of "coherence function," to grow from one maximum to the next along the line and, thus, enhances the conversion efficiency. Computer simulations of harmonic generation in LH NLTLs of different lengths (i.e., different numbers of stages) have shown that the conversion efficiency grows with the LH NLTL length, and finally saturates due to the transmission line loss. However, the 3-dB operating bandwidth decreases because $\phi$ becomes more sensitive to variation in the fundamental frequency with the increase of $n$. The maximum conversion efficiency generally takes place when $\left|\beta_{3}-3 \beta_{1}\right| \approx \pi$, and self-induced periodicity occurs.

Simulations have also shown that, under certain conditions, the self-induced periodicity may provide quasi-phase matching of the fundamental wave with some other $m$ th higher harmonic in a such a way that this $m$ th harmonic will dominate over other higher harmonics in the spectrum of the waveform at the LH NLTL output.

The conversion efficiency does not go to zero when the "coherence function" vanishes (when $|\phi|$ is $6 \pi$ or $4 \pi$ ) due to the variation of the phase shift per section along the line, imparted by the strong nonlinearity.

In summary, the analytical formulas obtained in Section III allow to us explain the basic features of the frequency dependence of the third harmonic conversion efficiency and will be useful for future optimization of the parameters of LH NLTLs. Simulation also predicts self-supported third harmonic generation in the case where $\left|\beta_{3}-3 \beta_{1}\right| \approx \pi$.

Furthermore, the periodicity (or discreteness) of the LH NLTL is essential for harmonic generation. The periodicity is inherent to realizable LH NLTLs. Harmonic generation in

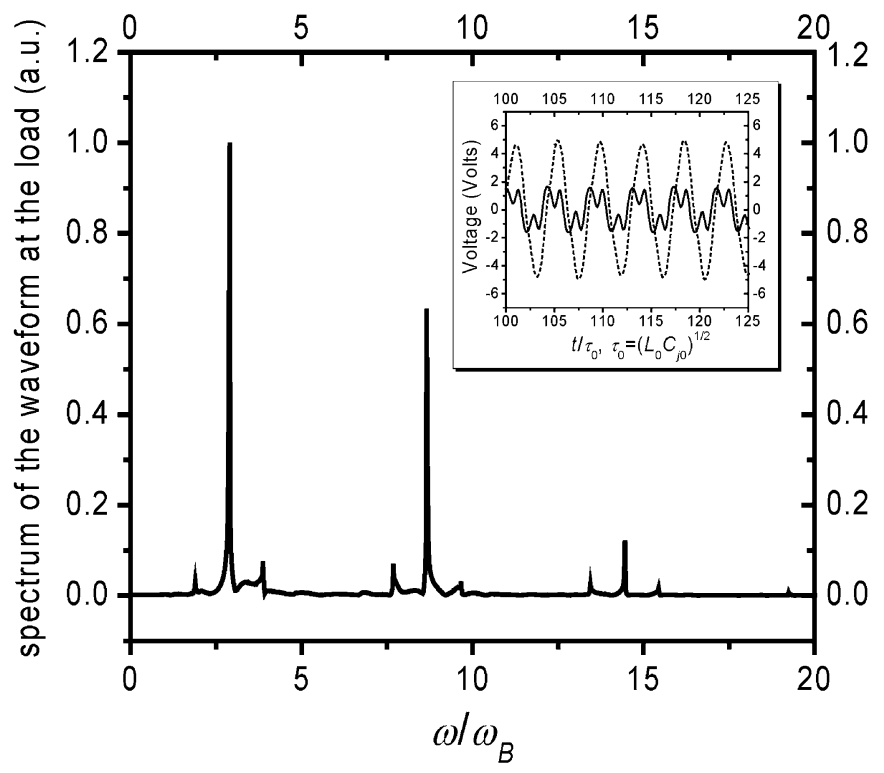

Fig. 5. Spectrum of the voltage waveform at the output load of an LH NLTL corresponding to the maximum of third harmonic conversion efficiency at the fundamental frequency $\omega_{\text {inp }} / \omega_{B}=2.89$ (from [16]). The original waveform is shown in the inset with solid line (dashed line shows voltage waveform at the input).

quasi-homogeneous LH materials, however, like those using arrays of metallic wires and split-ring resonators [1], [5] requires loading them periodically with nonlinear components.

Fig. 5 shows the spectrum of the voltage waveform at the load, corresponding to the maximum third harmonic conversion efficiency. The inset compares the original waveform with the waveform at the input. The fifth harmonic conversion efficiency is $\leq 1 \%$ so the power conversion into the third harmonic is very efficient (approximately 19\%), and generation of higher harmonics is suppressed.

For comparison, Fig. 3 shows the third harmonic generation efficiency in a dual RH NLTL of the same length (same number of sections) and based on the same varactor diodes. The third harmonic generation efficiency in the LH NLTL is higher (19\% versus 14\%), occurs at higher frequencies (maximum at $\omega / \omega_{B} \approx 2.9$ versus $\omega / \omega_{B} \approx 1.9$ ), and has a wider $3-\mathrm{dB}$ operating bandwidth (60\% versus $30 \%)$. The third harmonic conversion efficiency in the LH NLTL is of the same order as that which can be obtained using a conventional periodically loaded NLTL exhibiting normal dispersion (of the low-pass filter type) [18], [19], but the required transmission line length is shorter for the LH NLTL, thus, the per section efficiency is higher.

\section{Parametric Generation in LH NLTL}

The decrease in the third harmonic generation efficiency at the higher frequency boundary is related to other mechanisms of frequency conversion. One of these is the instability known as parametric generation of traveling waves [20]. It is known that parametric generation and amplification in dispersionless RH NLTLs is suppressed by shock-wave formation [21], [22]. The impossibility of shock waves in LH NLTLs gives rise to 


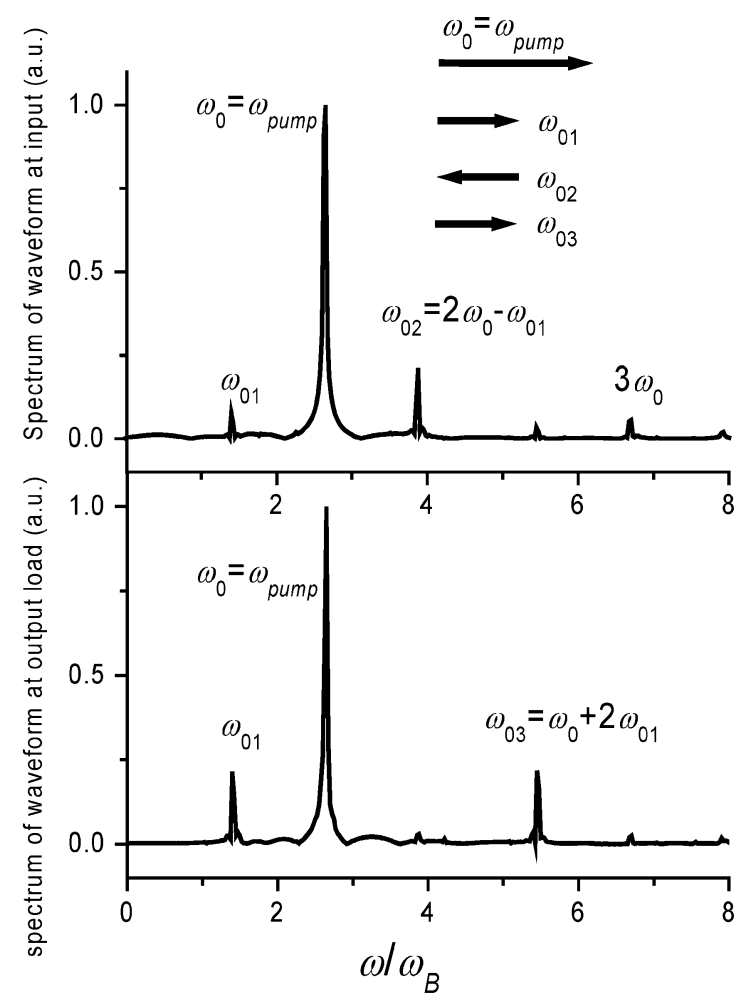

Fig. 6. Parametric generation: spectrum of the waveforms at the loads $R_{L} / Z_{0}=1$ connected at the input and output of a seven-section LH NLTL $\left(V_{\text {inp }}=2 \mathrm{~V}, \omega_{0} / \omega_{B}=2.64\right)$.

a variety of parametric processes that compete with harmonic generation.

The high-frequency fundamental (pump) wave with frequency $\omega_{0}$ and wavenumber $\beta_{0}$ generates two other waves having frequencies $\omega_{01}<\omega_{0}$ and $\omega_{02}=2 \omega_{0}-\omega_{01}$. This is illustrated in Fig. 6. Since $\omega_{0}-\omega_{01}=\omega_{02}-\omega_{0}$, we refer here to waves $\omega_{01}$ and $\omega_{02}$ as, respectively, Stokes and anti-Stokes waves by analogy with nonlinear optics [23]. The anti-Stokes wave at $\omega_{02}$ propagates in the opposite direction relative to the fundamental (pump) wave and the Stokes waves. A peak at $\omega_{02}$ in the spectrum of the input waveform can be seen, while it is absent at the output. Otherwise, the peak corresponding to the Stokes wave $\omega_{01}$ is larger at the output. The backward-propagating, parametrically generated anti-Stokes wave $\omega_{02}$ enables internal feedback and we, therefore, have a similar situation to backward wave parametric generation [20], [24]. The interaction of the fundamental wave $\omega_{0}$ and Stokes wave $\omega_{01}$ also lead to generation of a co-propagating wave at $\omega_{03}=\omega_{0}+2 \omega_{01}$.

The anomalous dispersion of $\mathrm{LH}$ medium allows for the phase matching of the fundamental and the parametrically generated waves so that the "coherence length" of the nonlinear parametric processes described above is large. The amplitude of the parametrically generated waves is predicted to grow with distance and they should compete with harmonic generation in the case of longer lines.

The waveform evolution in an LH NLTL has many similarities with the competition between resonant excitations and nonlinear parametric interactions recently discussed in nonlinear optics (see [25]).

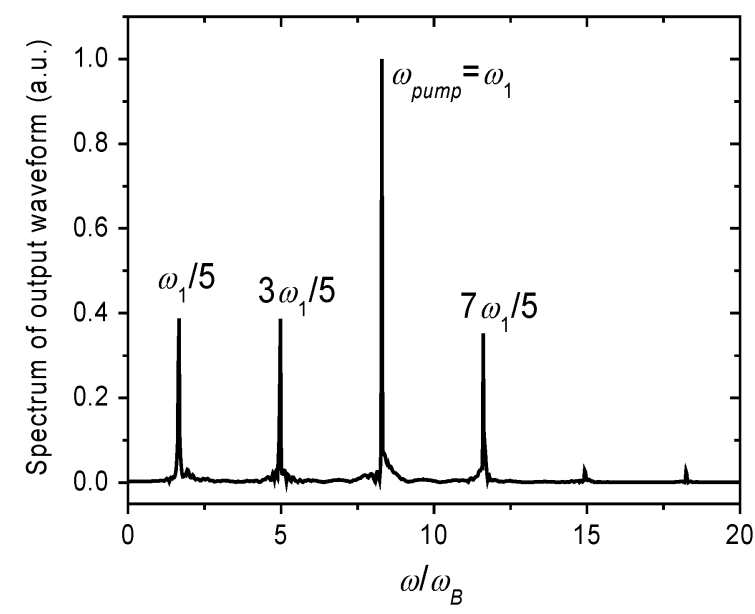

Fig. 7. Generation of fractional frequencies: spectrum of the waveform at the load $R_{L} / Z_{0}=1$ connected at the output of seven-section LH NLTL ( $V_{\text {inp }}=$ $\left.4.25 \mathrm{~V}, \omega_{1} / \omega_{B}=8.3\right)$.

\section{Generation of Fractional Frequencies and Other Regimes of Operation of LH NLTLS}

Another interesting possibility enabled by the interplay of anomalous dispersion and nonlinearity is the generation of fractional frequencies by high-frequency fundamental waves. This generation of fractional frequencies becomes possible when the frequency of the fundamental wave exceeds $3 \omega_{B}$. Anomalous dispersion allows phase matching of the fundamental wave with frequency $\omega_{1}$ and wavenumber $\beta_{1}$ with one of its fractional harmonics having frequency $\omega_{1 / m}=\omega_{1} / m$ and wavenumber $\beta_{1 / m}=m \beta_{1}$ ( $m$ is the integer number) so that the LH NLTL can function as a frequency divider. One can apply the analysis of Section III to show that the "coherence length" between the fundamental wave and a wave at some fractional frequency can be very long, as determined by

$$
N_{c} \sim \frac{2 \pi}{\left|\beta_{1 / m}-m \beta\right|} .
$$

Fig. 7 shows the spectrum of the voltage waveform at the output port of a seven-section LH NLTL. The fundamental wave $\omega_{1}$ effectively generates three other waves having frequencies $1 / 5 \omega_{1}, 3 / 5 \omega_{1}$, and $7 / 5 \omega_{1}$.

Under some conditions, LH NLTLs exhibit mixed behavior when parametrically generated waves start generating harmonics and vice versa. Parametric generation can be stimulated by harmonics of the fundamental wave resulting in a very complicated spectrum of the voltage waveform at the output. The upper inset in Fig. 4 gives an example of such behavior. One can find peaks related to parametric generation, higher harmonics, and fractional harmonics. Small peaks near the fundamental frequency and its higher harmonics (Fig. 5) also result from parametric generation. Thus, LH NLTLs can be operated in numerous regimes and switching between different regimes can be accomplished by changing the amplitude or frequency of the input signal.

Unlike in the case of generation of higher harmonics, the periodicity (discreteness) is not essential for either parametric generation or generation of fractional frequencies, both of which can also be observed in homogeneous LH nonlinear medium. 
Finally, we note that all the qualitative considerations presented here are valid for second harmonic generation in LH NLTLs periodically loaded with biased diodes exhibiting asymmetric capacitance-voltage characteristics. The preference given to third-order nonlinearity (symmetric capacitance-voltage characteristics) arises from the fact that two-dimensional (2-D) and three-dimensional (3-D) structures can be more easily realized if they do not require biasing.

\section{COMMENTS ON THE PHYSICAL IMPLEMENTATION OF LH NLTLs}

LH NLTLs can be fabricated by replacing the linear capacitors in the transmission-line LH medium discussed in [2], [6], and [7] with surface mount varactor diodes (e.g., by Agilent varactor diodes).

Depending on the amplitude and frequency of the input signal, hybrid LH NLTLs can be operated as frequency multipliers, parametric generators (amplifiers), or generators of fractional frequencies. The mode of operation is determined by the parameters of the LH NLTL and can be controlled electronically by varying the amplitude and frequency of the input drive signal. Mixed modes of operation are also possible under appropriate conditions.

Assuming $R_{L}$ to be $50 \Omega$, one can solve for the circuit parameters specified in Section IV-A, arriving at the value for line impedance $Z_{0}=62.5 \Omega$ and Bragg cutoff frequency $\omega_{B}=8 \mathrm{GHz}$. According to the results of the simulations in Section IV, the maximum of the third harmonic conversion efficiency should be achieved when a fundamental $3.7-\mathrm{GHz}$ 75-mW input signal is applied at the input of LH NLTL. The expected resulting power of the third harmonic delivered to the load is $14 \mathrm{~mW}$. We anticipate submitting a paper describing physical design of an LH NLTL and measurements of harmonic generation in the nearest future.

In addition to the higher conversion efficiency and broader operating frequency range mentioned in Section IV, the design of frequency multiplier based on LH NLTLs have some advantages over conventional periodically loaded RH NLTLs from a design perspective. They are more compact since the length of the section in practice is determined by the diode package size, and this size can be very small. They can also be low loss since less diodes are required to achieve the same value of conversion efficiency. Furthermore, at some parameters (when $\left|\beta_{1}\right|>\omega_{1} / c$ and $\left|\beta_{3}\right|<3 \omega_{1} / c$ and where $\omega_{1}$ is the frequency of the fundamental input signal and $c$ is the velocity of light in free space), LH NLTLs can be the waveguide for the fundamental input signal and a leaky-wave antenna [8], [9] for the generated third harmonic, thus, significantly simplifying the radiation of the generated power.

\section{CONCLUSION}

This paper has demonstrated a diversity of nonlinear wave phenomena in LH NLTLs, including harmonic generation and parametric generation of traveling waves, and their competition. Our simulations demonstrate efficient harmonic generation along LH NLTLs. Harmonic generation is possible over a significantly wider operating frequency range and at relatively higher frequencies in comparison with the dual conventional low-pass filter NLTL. Furthermore, LH NLTLs are predicted to have advantages from the design perspective since we have more freedom to optimize parameters, being much less restricted by the host waveguide structure than in the case of RH periodically loaded NLTLs. Extending these results for 1-D LH NLTL to higher dimensions would enable combining harmonic generation in LH NLTL media with focusing, due to the negative refractive index of 2-D or 3-D LH transmission-line media. This may lead to the development of highly efficient and powerful frequency multipliers. The parametric generation and amplification that generally accompany harmonic generation in LH NLTLs will be of interest for building "active" or "amplifying" super lenses based on LH nonlinear medium and provide a means to compensate for the inherent $\mathrm{LH}$ medium loss, which is a current challenge for existing LH materials.

\section{ACKNOWLEDGMENT}

The authors thank D. Yavuz for his useful comments, and C. Paulson, A. Bettermann, and A. Karbassi, for careful reading and editing of this paper's manuscript.

\section{REFERENCES}

[1] D. R. Smith, W. J. Padilla, D. C. Vier, S. C. Nemat-Nasser, and S. Schultz, "Composite medium with simultaneously negative permeability and permittivity," Phys. Rev. Lett., vol. 84, pp. 4184-4187, May 2000.

[2] G. V. Eleftheriades, A. K. Iyer, and P. C. Kremer, "Planar negative refractive index media using periodically $L-C$ loaded transmission lines," IEEE Trans. Microw. Theory Tech., vol. 50, no. 12, pp. 2702-2712, Dec. 2002.

[3] A. Grbic and G. V. Eleftheriades, "Overcoming the diffraction limit with a planar left-handed transmission-line lens," Phys. Rev. Lett., vol. 92, no. 11, p. 117 403-1, Mar. 19, 2004.

[4] V. G. Veselago, "The electrodynamics of substances with simultaneously negative values of $\varepsilon$ and $\mu$," Sov. Phys.-Usp., vol. 10, no. 4, pp. 509-514, Jan.-Feb. 1968.

[5] R. A. Shelby, D. R. Smith, and S. Schultz, "Experimental verification of a negative index of refraction," Science, vol. 292, pp. 77-79, Apr. 2001.

[6] M. Anioniades and G. V. Eleftheriades, "Compact linear lead/lag metamaterial phase shifters for broadband applications," IEEE Antennas Wireless Propag. Lett., vol. 2, no. 7, pp. 103-106, 2003.

[7] C. Caloz, A. Sanada, and T. Itoh, "A novel composite right-/left-handed coupled-line directional coupler with arbitrary coupling level and broad bandwidth," IEEE Trans. Microw. Theory Tech., vol. 52, no. 3, pp. 980-992, Mar. 2004.

[8] L. Liu, C. Caloz, and T. Itoh, "Dominant mode leaky-wave antenna with backfire-to-endfire scanning capability," Electron. Lett., vol. 38, no. 23, pp. 1414-1416, Nov. 2002.

[9] A. Grbic and G. V. Eleftheriades, "Experimental verification of backward-wave radiation from a negative refractive index metamaterial," $J$. Appl. Phys., vol. 92, no. 10, pp. 5930-5935, Nov. 15, 2002.

[10] A. A. Zharov, I. V. Shadrivov, and Y. S. Kivshar, "Nonlinear properties of left-handed metamaterials," Phys. Rev. Lett., vol. 91, no. 3, p. 037 401-1, Jul. 18, 2003.

[11] C. Caloz, I. H. Lin, and T. Itoh, "Characteristics and potential applications of nonlinear left-handed transmission lines," Microwave Opt. Technol. Lett., vol. 40, no. 6, pp. 471-473, Mar. 2004.

[12] A. M. Belyantsev and A. B. Kozyrev, "RF oscillation generated by an electromagnetic shock wave in coupled transmission lines with anomalous and normal dispersion," Tech. Phys., vol. 46, no. 7, pp. 864-867, 2001. 
[13] A. B. Kozyrev, "The structure of a shock electromagnetic wave synchronous with several waves propagating in coupled transmission lines with different types of dispersion," Tech. Phys., vol. 47, no. 2, pp. 272-274, 2002.

[14] A. M. Belyantsev and A. B. Kozyrev, "Reversed Doppler effect under reflection from a shock electromagnetic wave," Tech. Phys., vol. 47, no. 11, pp. 1477-1480, 2002.

[15] I. V. Shadrivov, A. A. Sukhorukov, Y. S. Kivshar, A. A. Zharov, A. D. Boardman, and P. Egan, "Nonlinear surface waves in left-handed materials," Phys. Rev. E, Stat. Phys. Plasmas Fluids Relat. Interdiscip. Top., vol. 69, p. 16617-1, Jan. 2004.

[16] A. B. Kozyrev and D. van der Weide, "Nonlinear transmission lines in left-handed media," in IEEE MTT-S Int. Microwave Symp. Dig., 2004, pp. 317-320.

[17] K. S. Champlin and D. R. Singh, "Small-signal second-harmonic generation by a nonlinear transmission line," IEEE Trans. Microw. Theory Tech., vol. MTT-34, no. 3, pp. 351-353, Mar. 1986.

[18] J.-M. Duchamp, P. Ferrari, M. Fernandez, A. Jrad, X. Melique, J. Tao, S. Arscott, D. Lippens, and R. G. Harrison, "Comparison of fully distributed and periodically loaded nonlinear transmission lines," IEEE Trans. Microw. Theory Tech., vol. 51, no. 4, pp. 1105-1116, Apr. 2003.

[19] M. J. W. Rodwell, S. T. Allen, R. Y. Yu, M. G. Case, U. Bhattacharya, M Reddy, E. Carman, M. Kamegawa, Y. Konishi, J. Pusl, R. Pullela, and J. Esch, "Active and nonlinear wave propagation devices in ultrafast electronics and optoelectronics," Proc. IEEE, vol. 82, no. 7, pp. 1035-1059, Jul. 1994.

[20] A. S. Gorshkov, G. A. Lyakhov, K. I. Voliak, and L. A. Yarovoi, "Parametric generation in anomalously dispersive media," Physica D, vol. 122, pp. 161-177, 1998.

[21] R. Landauer, "Shock waves in nonlinear transmission lines and their effect on parametric amplification," IBM J., vol. 4, pp. 391-401, 1960.

$[22]$ - "Parametric amplification along nonlinear transmission line," $J$. Appl. Phys., vol. 31, no. 3, pp. 479-484, Mar. 1960.

[23] A. Yariv, Quantum Electronics. New York: Wiley, 1988.

[24] S. E. Harris, "Proposed backward wave oscillation in the infrared," Appl. Phys. Lett., vol. 9, no. 3, pp. 114-116, Aug. 1, 1966.

[25] R. Frey, "Suppression of the medium excitation in resonant nonlinear optics," Opt. Commun., vol. 89, no. 5-6, pp. 441-446, May 15, 1992.

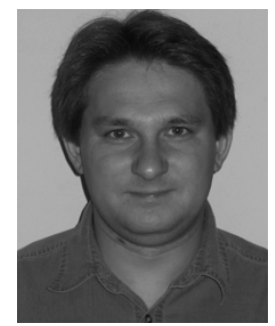

Alexander B. Kozyrev was born in Gorky (now Nizhny Novgorod), Russia, in 1971. He received the Diploma degree (with distinction) in radiophysics and electronics from Nizhny Novgorod State University, Nizhny Novgorod, Russia, in 1993 and the Ph.D. degree (Candidate of Science degree in physics and mathematics) from the Institute for Physics of Microstructures, Russian Academy of Sciences (RAS), Nizhny Novgorod, Russia, in 2001

From 1993 to 1994, he was with the Institute of Applied Physics, RAS. In 1994, he joined the Institute for Physics of Microstructures, RAS. Since 2003, he has been a Research Associate with the University of Wisconsin-Madison. His research interests include wave-propagation phenomena in NLTLs and their applications in microwave electronics, LH metamaterials, ultrafast phenomena in semiconductors, and semiconductor heterostructures.

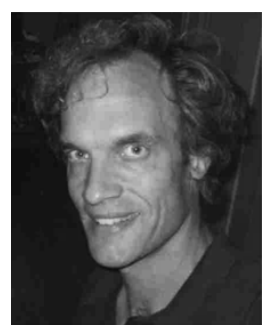

Daniel W. van der Weide (S'86-M'86) received the B.S.E.E. degree from the University of Iowa, Iowa City, in 1987, and the Master's and Ph.D. degrees in electrical engineering from Stanford University, Stanford, CA, in 1989 and 1993, respectively.

He held summer positions with the Lawrence-Livermore National Laboratory and Hewlett-Packard, and full-time positions with Motorola as an Engineer and the Watkins-Johnson Company as a Member of the Technical Staff. From 1993 to 1995, he was a Post-Doctoral Researcher with the Max-Planck-Institut für Festkörperforschung (Solid State Research), Stuttgart, Germany, after which he joined the Department of Electrical and Computer Engineering, University of Delaware, as an Assistant and Associate Professor and Director of the Center for Nanomachined Surfaces. In 1999, he joined the Department of Electrical and Computer Engineering, University of Wisconsin-Madison, as an Associate Professor. From 2002 to 2004, he was a University of Wisconsin Vilas Associate. He was the Principal Investigator on a 2003 Air Force Office of Scientific Research (AFOSR) Multiuniversity Research Initiative (MURI) overseen by Lt. Col. G. Pomrenke entitled, "Nanoprobe Tools for Molecular Spectroscopy and Control." His current research involves ultrafast electronics, 1-D electron systems, and the application of high-frequency techniques in biotechnology.

Dr. van der Weide was the recipient of the National Science Foundation (NSF) CAREER and PECASE Awards in 1997 and the Office of Naval Research (ONR) Young Investigator Program Award in 1998. 